TITLE:

\title{
Photonic band structure calculations of two-dimensional Archimedean tiling patterns
}

$\operatorname{AUTHOR}(\mathrm{S})$ :

Ueda, K; Dotera, T; Gemma, T

\section{CITATION:}

Ueda, K ... [et al]. Photonic band structure calculations of two-

dimensional Archimedean tiling patterns. PHYSICAL REVIEW B 2007, 75(19): 195122.

ISSUE DATE:

2007-05

URL:

http://hdl.handle.net/2433/50200

RIGHT:

Copyright 2007 American Physical Society 
PHYSICAL REVIEW B 75, 195122 (2007)

\title{
Photonic band structure calculations of two-dimensional Archimedean tiling patterns
}

\author{
Kazunari Ueda, ${ }^{1}$ Tomonari Dotera, ${ }^{1}$ and Tohru Gemma ${ }^{2}$ \\ ${ }^{1}$ Department of Polymer Chemistry, Kyoto University, Katsura, Nishikyo-ku, Kyoto 615-8510, Japan \\ ${ }^{2}$ Graduate School of Arts and Sciences, University of Tokyo, Komaba, Meguro-ku, Tokyo 153-8902, Japan
}

(Received 20 July 2006; revised manuscript received 28 March 2007; published 22 May 2007)

\begin{abstract}
We present a study of photonic band structures of two-dimensional Archimedean tiling patterns. The tilings we have investigated are $\left(4.8^{2}\right),\left(6^{3}\right),(4.6 .12)$, and $\left(3^{2} .4 .3 .4\right)$, which have been discovered computationally and experimentally in self-assembled microphase separation of $A B C$ star block terpolymer systems. Using plane-wave method, we have calculated eigenvalue equations for various combinations of dielectric contrast on the complex patterns. We demonstrate the existence of complete photonic band gaps in the (4.6.12) structure. Furthermore, we find that complete photonic bands readily open in the $\left(3^{2} .4 .3 .4\right)$ structures in the same way as in dodecagonal quasicrystals. Complex tilings open up a way to construct photonic crystals.
\end{abstract}

DOI: 10.1103/PhysRevB.75.195122

PACS number(s): 78.67.-n, 42.70.Qs, 83.80.Uv

\section{INTRODUCTION}

Archimedean tilings depicted by Kepler in Harmonices Mundi II (1619) are regular patterns of polygonal tessellation in plane by using regular polygons. ${ }^{1}$ According to Kepler, they are congruent because only one type of vertices is permitted in each tiling. It is known that only 11 kinds of Archimedean tilings illustrated in Fig. 1 can fill the whole plane without gaps. Here, a set of integers $\left(n_{1} \cdot n_{2} \cdot n_{3}, \cdots\right)$ denotes a tiling of a vertex type in the way that $n_{1}$-gon, $n_{2}$-gon, and $n_{3^{-}}$gon, $\ldots$, meet consecutively on each vertex. The symbol $\left(3^{2} .4 .3 .4\right)$, for instance, represents a tiling in which two equilateral triangles, a square, an equilateral triangle, and a square gather edge-to-edge around a vertex.

Recently, several Archimedean tiling structures denoted by $\left(6^{3}\right),\left(4.8^{2}\right),(4.6 .12)$, and $\left(3^{2} .4 .3 .4\right)$ were discovered in the self-organized structures of synthetic polymer systems. ${ }^{2-9}$ Interestingly, the lattice constants of the structures can reach the wavelength of visible light. Hence, they may open the possibility of constructing novel photonic band gap (PBG) devices, such as waveguides or dielectric mirrors, where the propagation of electromagnetic waves or the spontaneous emission of light is forbiddden. ${ }^{10-19}$ In this paper, we present photonic band structures of the four Archimedean tiling patterns accessible in the self-assembling structures.

To generate such mesoscopic patterns, much attention has been paid to block copolymer systems. ${ }^{20-24}$ Intramolecular segregation of block copolymers consisting of different polymers covalently linked together gives rise to microphase separations producing periodic morphologies: lamellar, cocontinuous, hexagonally cylindrical, and bcc spherical structures are well known. Concerning optical properties, triply periodic cocontinuous structures such as double-diamond and gyroid structures have been extensively investigated by several researchers. ${ }^{25-30}$

The focus of the present paper is two-dimensionally complex patterns produced by $A B C$ star block copolymers (terpolymers) consisting of chemically distinct three polymers linked at one junction. Their melts can form two-dimensional tiling patterns, precisely, polygonal cylindrical phases whose sections are the Archimedean tilings. If the interactions between $A B C$ polymer components are equally strong, only $\left(6^{3}\right),\left(4.8^{2}\right)$, and (4.6.12) belonging to the single junction class $^{31}$ (SJC) can be obtained as direct patterns, ${ }^{5}$ where each polygon in the Archimedean tiling directly corresponds to each polymeric microdomain. It is firstly because only three polygons corresponding to $A B C$ microdomains should meet on a vertex, and secondly because only even polygons should appear, which fact is called even polygon theorem. ${ }^{2,32}$ Very recently, an indirect skeleton tiling $\left(3^{2} .4 .3 .4\right)$ has been obtained. ${ }^{6}$ The tiling is more complex than the SJC; however, the skeleton structure is the $\left(3^{2} .4 .3 .4\right)$ Archimedean tiling. Therefore, we consider three direct tilings, $\left(6^{3}\right),\left(4.8^{2}\right)$, and (4.6.12), and one skeleton tiling, $\left(3^{2} .4 .3 .4\right)$, in the present paper.

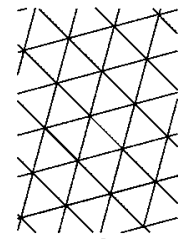

$\left(3^{6}\right)$

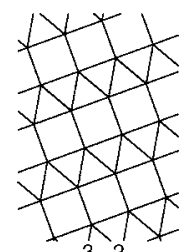

$\left(3^{3} \cdot 4^{2}\right)$

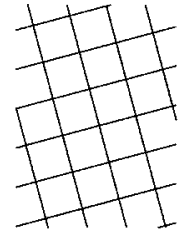

$\left(4^{4}\right)$

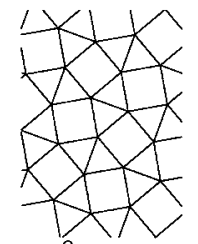

$\left(3^{2} \cdot 4.3 .4\right)$

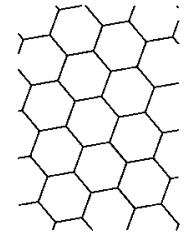

$\left(6^{3}\right)$

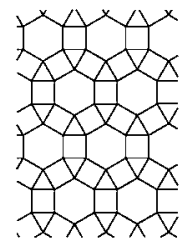

(3.4.6.4)

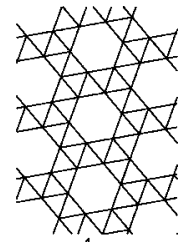

$\left(3^{4} .6\right)$

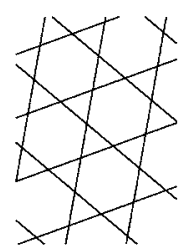

(3.6.3.6)

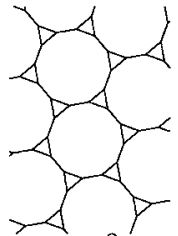

$\left(3.12^{2}\right)$

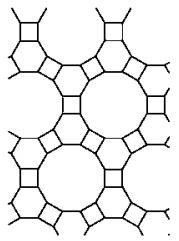

(4.6.12)

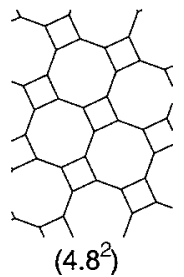

FIG. 1. Archimedean tilings: A set of integers $\left(n_{1} \cdot n_{2} \cdot n_{3}, \cdots\right)$ denotes a tiling of a vertex type in the way that $n_{1}$-gon, $n_{2}$-gon, and $n_{3}$-gon, $\ldots$, meet consecutively on each vertex. Superscripts are employed to abbreviate when possible. There exist only 11 types of tiling by regular polygons, where all vertices are of the same type. 


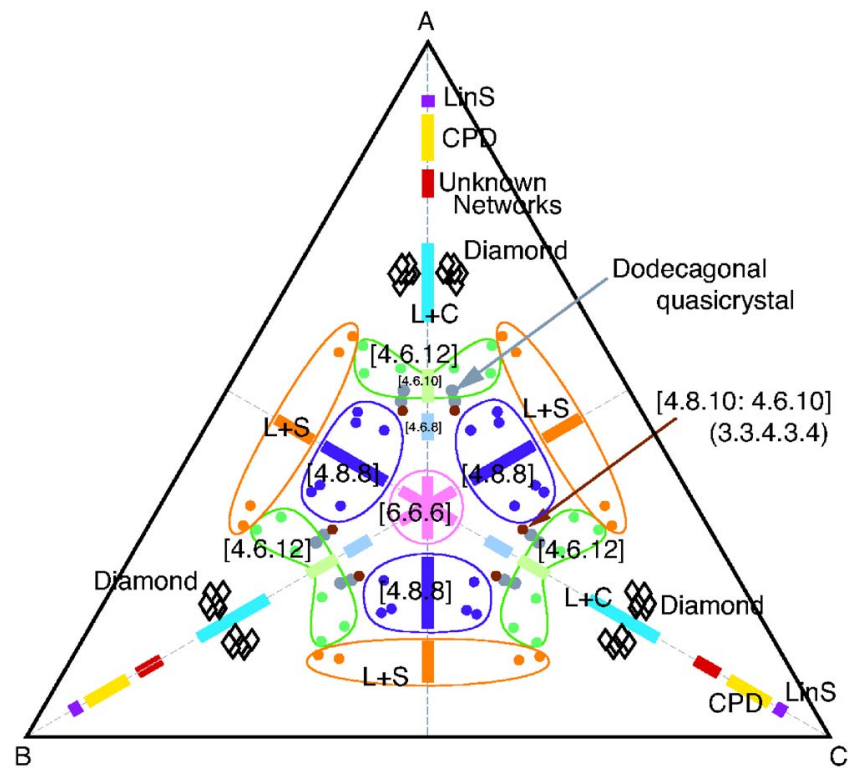

FIG. 2. (Color online) MC simulation results: Phase diagram as functions of arm-length ratio of $A B C$ star terpolymer system with symmetric interactions between three components. Morphologies in the central region are five cylindrical structures: $\left(4.8^{2}\right),\left(6^{3}\right)$, $\left(3^{2} .4 .3 .4\right)$, dodecagonal quasicrystal, and (4.6.12). Threedimensional structures are perforated layer (PL), lamella+cylinder $(\mathrm{L}+\mathrm{C})$, single diamond (Diamond), columnar piled disk (CPD), lamella-in-sphere (L-in-S), and lamella+sphere $(\mathrm{L}+\mathrm{S})$. See details in Ref. 2.

It is worthwhile to mention that dodecagonal quasicrystals ${ }^{33-36}$ are thought to be promising candidates for PBG structures because of their high degree of rotational symmetry. ${ }^{37-44}$ It was suggested that a complete PBG opens with low dielectric contrasts in a dodecagonal structure. ${ }^{37}$ Although the two-dimensional (2D) space group is $\mathrm{p} 4 \mathrm{gm}$, the $\left(3^{2} .4 .3 .4\right)$ tiling produces 12 -fold-like Fourier peaks, ${ }^{45}$ and it is an approximant of the dodecagonal quasicrystal. ${ }^{35}$ Therefore, the band structure of $\left(3^{2} .4 .3 .4\right)$ is worth considering. In this direction, a systematic study has been reported. ${ }^{38}$ Finally, we should mention that a Monte Carlo (MC) simulation $^{45}$ and a mean-field theory ${ }^{46}$ of $A B C$ star terpolymers suggest a soft and mesoscopic self-organized dodecagonal quasicrystal, which has been realized recently. ${ }^{47}$

The organization of paper is as follows. In Sec. II, we provide an Archimedean tiling phase diagram of $A B C$ star terpolymers obtained by MC simulations. We explain the MC simulation method and present some results. In Sec. III, we elucidate how to construct tiling patterns with the arbitrary component ratio of $A B C$ species by a geometric operation in terms of minimal fundamental triangles. The method of photonic band calculations for both $E$ and $H$ polarizations is provided in Sec. IV. We have used plane-wave expansion method. ${ }^{13-18}$ In Sec. V, the main results of the paper are presented. Sec. VI is devoted to discussion and summary.

\section{MONTE CARLO SIMULATION OF ABC STAR TERPOLYMERS}

In this section, we present a phase diagram and an example of the $\left(3^{2} .4 .3 .4\right)$ structure obtained in our MC simu-
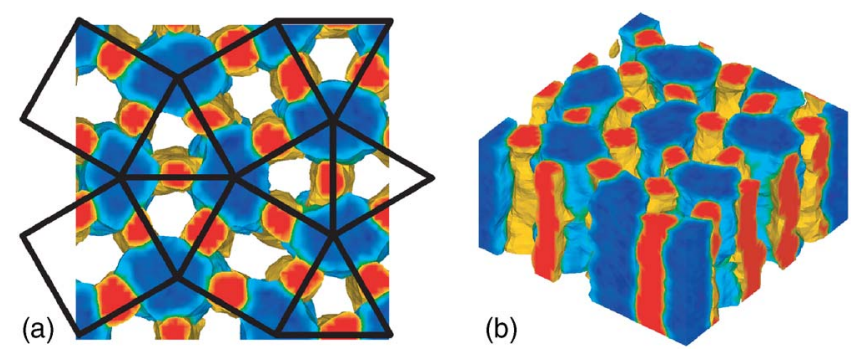

FIG. 3. (Color online) MC simulation result of $\left(3^{2} .4 .3 .4\right)$ by $A 9 B 7 C 14$ star-shaped terpolymers in a box with $25 \times 48 \times 48$. Small domains represent $B$, large ones are $C$, and $A$ is transparent. (a) Top view. The skeleton $\left(3^{2} .4 .3 .4\right)$ net is superimposed. (b) Cylindrical structure is shown. Both pictures represent the volume rendering of averaged densities over $10^{3} \mathrm{MC}$ steps at $\beta=0.071$.

lations. Since details of the method were described in Refs. 2 and 3 , we briefly explain it.

The method is a simple extension of the bead-and-bond lattice polymer MC method called "diagonal bond method." A bead occupies only one lattice point to ensure excluded volume interactions as usual; the difference is that the bond length can be $1, \sqrt{2}$, or $\sqrt{3}$ in the unit of lattice spacing. One $A B C$ star block copolymer consists of $N_{A} A$-type beads, $N_{B}$ $B$-type beads, $N_{C} C$-type beads, and one $Y$-type bead (junction point), which are connected by $N-1$ bonds, where $N$ $=N_{A}+N_{B}+N_{C}+1$.

To represent energetics that drives the system to microphase separation, unit contact energies are imposed only between pairs of different species within the body diagonal distance $\sqrt{3}$. We consider the Hamiltonian as $\sum \epsilon_{i j}$, where $\epsilon_{i j}=1$ when $i \neq j$, and $i$ and $j$ stand for $A, B$, or $C$. The MC procedure is the following: We select one bead randomly and choose a trial move randomly out of possible moves; if the trial is a vacancy, we determine move or not according to the Metropolis algorithm.

Cubic boxes up to $L^{3}$ with $L=30-64$, or quasi-twodimensional boxes with $L_{x}=L_{y}=48-128$ and $L_{z}=10-25$, subjected to periodic boundary conditions have been used. The occupation ratio of beads in the lattice points is 0.75 . The system is prepared as totally randomized at the infinite temperature, and then quenched at $\beta=1 / k_{B} T=0.07-0.11$ to wait ordering, where $k_{B}$ is the Boltzmann constant and $T$ is absolute temperature.

The phase diagram obtained from simulations is illustrated in Fig. 2. The line with the condition that $N_{A}=N_{B}$ has been seen in Ref. 2. Two-dimensional polygonal cylindrical patterns occupy the central region of the triangular phase diagram. Figure 3 depicts the result of a unit cell $\left(3^{2} .4 .3 .4\right)$ structure. A large-scale quasi-two-dimensional simulation result has been demonstrated in Ref. 45.

\section{GEOMETRY OF TILINGS}

\section{A. Single junction class: $\left(6^{3}\right),\left(4.8^{2}\right)$, and (4.6.12)}

Evidently, in real microphase separations, the area ratios of $A B C$ components are not the same as those of the Archimedean tilings that are by definition composed of regu- 

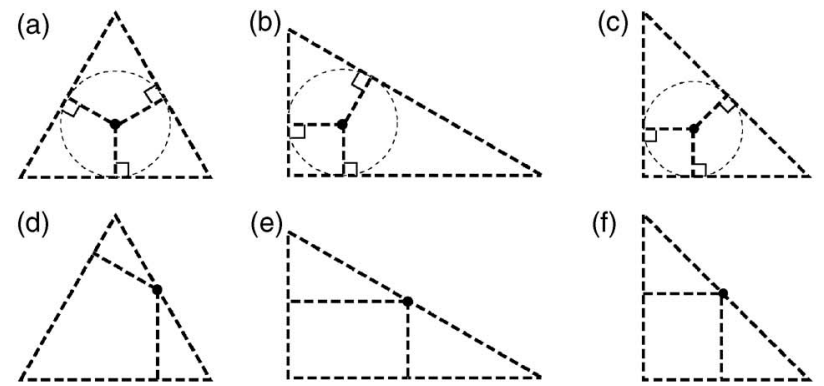

(e)

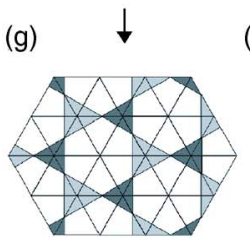

(h)

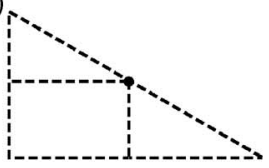

(f)
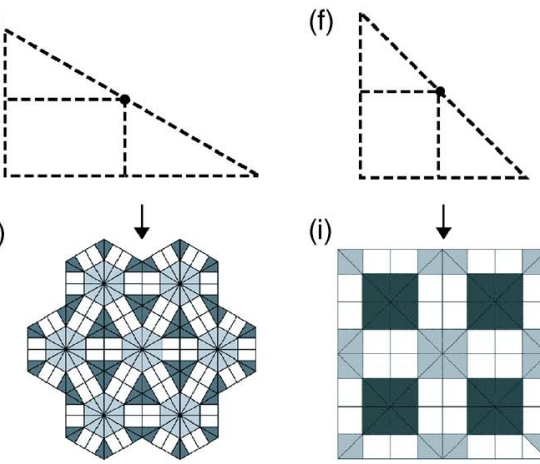

(j)

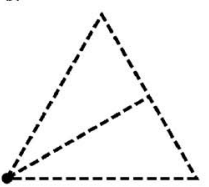

(k)

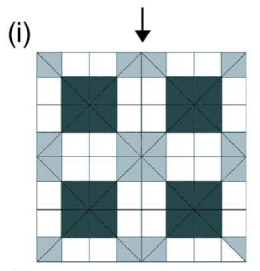

(I)

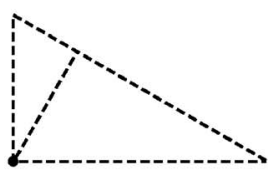

(n)
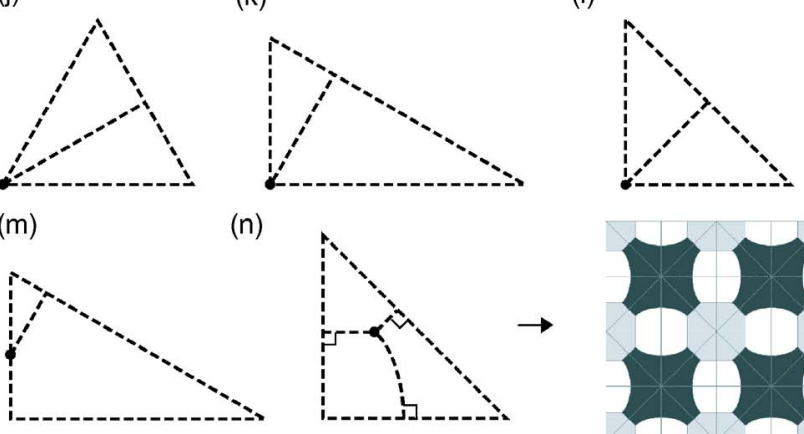

(m)

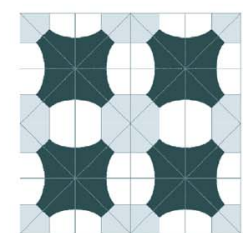

FIG. 4. (Color online) Minimal fundamental triangles of (a) $\left(6^{3}\right)$, (b) (4.6.12), and (c) $\left(4.8^{2}\right)$ Archimedean tilings, which belong to the single junction class. Limiting cases (d)-(f) and the resulting tiling (g) (3.6.3.6), (h) (3.4.6.4), and (i) $\left(4^{4}\right)$, respectively. Other limiting cases $(\mathrm{j})\left(3^{6}\right)$, (k) (3.6.3.6), (1) $\left(4^{4}\right)$, and (m) $\left(3.12^{2}\right)$. (n) St. Andrew's cross having curved polygons from the minimal triangle of $\left(4.8^{2}\right)$.

lar polygons. Furthermore, interactions between components affect the length and the shape of polygon boundaries. In this section, we explain how to construct the tiling structures for photonic band calculations that are obtained in experiments. First, we will use the symbols $\left(n_{1} . n_{2} . n_{3} . \cdots\right)$ for tilings consisting of nonregular or curved polygons as well: $n_{i}$ means a polygon surrounded by $n_{i}$ polygons with different chemical species, and then $\left(n_{1} \cdot n_{2} \cdot n_{3} . \cdots\right)$ means a tiling composed of vertices at which $n_{1}$-gon, $n_{2}$-gon, $n_{3}$-gon, and so on meet.

The single junction class (SJC) of the Archimedean tilings, which corresponds to three types of minimal fundamental triangles, ${ }^{2}$ plays a quite important role. Notice the three minimal fundamental triangles in Figs. 4(a)-4(c). The minimal fundamental triangle tiles the whole plane by mirror symmetric operations with respect to its edges. In mathematical terms, (a), (b), and (c) are associated with $A_{2}, G_{2}$, and $B_{2}$ root systems, respectively. The shape of the minimal fundamental triangle is specific to the phase type and is independent of the area ratio of each component. As in Figs. 4(a)-4(c), if the vertex (solid circle) called junction here is the incenter of the triangle and if three lines (emanating from the junction) that represent domain boundaries are straight

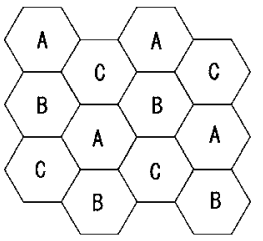

(a)

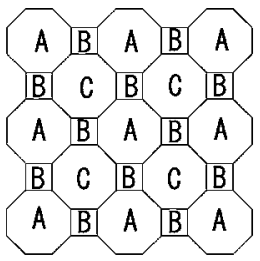

(b)

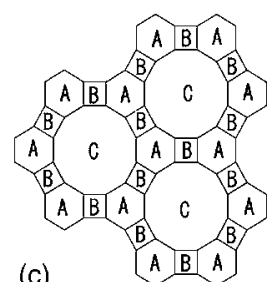

(c)
FIG. 5. Area ratios of photonic crystals: (a) $A: B: C=1: 1: 1$ in the $\left(6^{3}\right)$ structure, (b) $A: B: C=2: 1: 2$ in the $\left(4.8^{2}\right)$ structure, and (c) $A: B: C=1: 1: 2$ in the (4.6.12) structure. These ratios are used in photonic band calculations.

and perpendiculars to the edges of the triangle, then exact Archimedean tilings (a) $\left(6^{3}\right)$, (b) (4.6.12), and (c) $\left(4.8^{2}\right)$ composed of regular polygons are obtained.

To obtain a tiling corresponding to an experiment, put a junction on an appropriate position inside a fundamental triangle and draw three straight or curved lines to three edges such that the lines are perpendicular to the edges at the edges of the triangle (mirror planes). Our SJC photonic crystals we consider here are depicted in Fig. 5. The ratios of components are $A: B: C=1: 1: 1$ for the $\left(6^{3}\right)$ structure, $A: B: C$ $=2: 1: 2$ for the $\left(4.8^{2}\right)$ structure, and $A: B: C=1: 1: 2$ for the (4.6.12) structure, which are typical ratios in numerical experiments. We assume that domain boundaries are straight lines in our calculations. Dielectric contrasts will be described later.

Occasionally, it has been stated that observed tiling types are designated different from the SJC. In view of symmetry, they can be unified into the classification scheme of the SJC. Let us consider limiting cases that the junction is on an edge or a vertex of a minimal fundamental triangle. For example, we can construct (h) a (3.4.6.4) tiling from (e), which corresponds to the structure that has been obtained by Sioula $e t$ al. ${ }^{9}$ In this case, repulsion between triangular and hexagonal regions are stronger than others. There is a number of cases, which is summarized in Table I and Figs. 4(d)-4(n). Finally, we can employ curved domain boundaries instead of straight lines. For example, in terms of the minimal triangle of $\left(4.8^{2}\right)$, we are able to construct a pattern (n) called St. Andrew's cross by Yamauchi et al. ${ }^{8}$

TABLE I. Minimal fundamental triangle type denoted by one of the single junction class (SJC) and their limiting cases [Figs. 4(d) $-4(\mathrm{f})$ and 4(j) $-4(\mathrm{~m})$ ] and resulting Archimedean tilings.

\begin{tabular}{lccc}
\hline \hline & SJC & $\rightarrow$ Tiling & \\
\hline (d) & $\left(6^{3}\right)$ & $(3.6 .3 .6)$ & $(\mathrm{g})$ \\
$(\mathrm{e})$ & $(4.6 .12)$ & $(3.4 .6 .4)$ & $(\mathrm{h})$ \\
$(\mathrm{f})$ & $\left(4.8^{2}\right)$ & $\left(4^{4}\right)$ & $(\mathrm{i})$ \\
$(\mathrm{j})$ & $\left(6^{3}\right)$ & $\left(3^{6}\right)$ & \\
$(\mathrm{k})$ & $(4.6 .12)$ & $(3.6 .3 .6)$ & \\
$(\mathrm{l})$ & $\left(4.8^{2}\right)$ & $\left(4^{4}\right)$ & \\
$(\mathrm{m})$ & $(4.6 .12)$ & $\left(3.12^{2}\right)$ & \\
(n) & $\left(4.8^{2}\right)$ & $\left(4.8^{2}\right)$ & St. Andrew's cross \\
\hline \hline
\end{tabular}




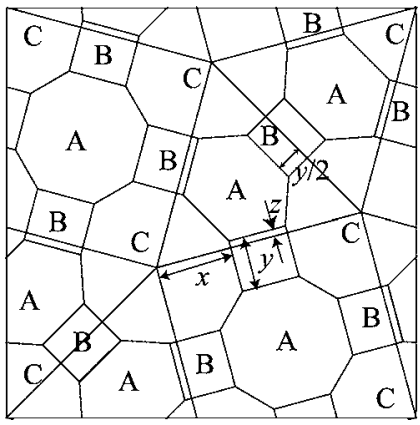

FIG. 6. Unit cell of the $\left(3^{2} .4 .3 .4\right)$ structure. The area ratio calculated in this paper is $A: B: C=9: 7: 14$ or $9: 7: 16$. The twodimensional space group is $p 4 \mathrm{gm}$.

\section{B. Skeleton tiling $\left(3^{2}\right.$.4.3.4)}

The construction of the $\left(3^{2} .4 .3 .4\right)$ structure is a nontrivial problem. Notice the unit cell of the $\left(3^{2}\right.$.4.3.4) structure (Fig. 6). There are two squares and four equilateral triangles in the unit cell. The plane group is $p 4 \mathrm{gm}$ : The center of a square has $C_{4}$ point symmetry and there are mirror planes passing through consecutive triangles. ${ }^{6}$ Therefore, we rationally impose the $C_{4}$ symmetry on squares and the mirror symmetry on triangles. Furthermore, we assume three additional requirements for simplicity: (1) Squares have $C_{4 v}$ symmetry inside them, (2) two types of $B$ rectangles are of the same shape, and (3) all domain boundaries are straight lines. Consequently, the positions of domains can be assigned by only three parameters $x, y$, and $z$ as shown in Fig. 6 .

Physically, the density of junctions of molecules on all vertices seems to be the same. ${ }^{2}$ If so, the area ratio of an $A$ hexagon to an $A$ octagon must be $6: 8$. The ratios of $A, B$, and $C$ are known. Using these three relations, we can determine $x, y$, and $z$. The area ratio we have calculated in this paper is $A: B: C=9: 7: 14$ or $9: 7: 16$.

\section{METHOD OF PHOTONIC BAND CALCULATION}

In this section, we outline the calculation method of photonic band structures for two-dimensional crystalline structures and show the accuracy of our computation. We employ the plane-wave expansion method.

\section{A. Plane-wave expansion method}

Since the dielectric structure is uniform in the $z$ direction, the relative dielectric constant $\varepsilon$ is a function of $x$ and $y$ denoted by $\mathbf{x}: \varepsilon=\varepsilon(\mathbf{x})$. The two-dimensional Bravais lattice is spanned by two primitive vectors $\mathbf{a}_{1}$ and $\mathbf{a}_{2}$. The dielectric structure satisfies the periodic boundary condition

$$
\varepsilon\left(\mathbf{x}+\mathbf{a}_{i}\right)=\varepsilon(\mathbf{x}) .
$$

The reciprocal lattice vectors $\mathbf{b}_{1}$ and $\mathbf{b}_{2}$ are defined by

$$
\mathbf{a}_{i} \cdot \mathbf{b}_{j}=2 \pi \delta_{i j},
$$

where $\delta_{i j}$ is the Kronecker delta symbol.

To solve the Maxwell equations, we expand $1 / \varepsilon$ by the Fourier series:

$$
\frac{1}{\varepsilon(\mathbf{x})}=\sum_{\mathbf{G}} \kappa(\mathbf{G}) e^{i \mathbf{G} \cdot \mathbf{x}},
$$

where the sum is taken over every reciprocal lattice vector $\mathbf{G}$, which is a linear combination of $\mathbf{b}_{1}$ and $\mathbf{b}_{2}$ :

$$
\mathbf{G}=l_{1} \mathbf{b}_{1}+l_{2} \mathbf{b}_{2},
$$

where $l_{1}$ and $l_{2}$ are integers. The Fourier coefficient is expressed by

$$
\kappa(\mathbf{G})=\frac{1}{S_{0}} \int_{S_{0}} d \mathbf{x} \frac{1}{\varepsilon(\mathbf{x})} e^{-i \mathbf{G} \cdot \mathbf{x}},
$$

where $S_{0}$ denotes the area of the unit cell.

We assume that the magnetic permeability of the photonic crystals is equal to that in free space, $\mu_{0}: \mathbf{B}(\mathbf{r}, t)=\mu_{0} \mathbf{H}(\mathbf{r}, t)$. Maxwell equations become

$$
\begin{gathered}
\nabla \times \mathbf{E}(\mathbf{r}, t)=-\mu_{0} \frac{\partial}{\partial t} \mathbf{H}(\mathbf{r}, t), \\
\nabla \times \mathbf{H}(\mathbf{r}, t)=\varepsilon_{0} \varepsilon(\mathbf{r}) \frac{\partial}{\partial t} \mathbf{D}(\mathbf{r}, t),
\end{gathered}
$$

where $\mathbf{E}$ and $\mathbf{H}$ are the electric and magnetic fields, and $\mathbf{D}$ and $\mathbf{B}$ are the displacement and magnetic induction fields, respectively. $\varepsilon_{0}$ is the dielectric constant in free space.

The electromagnetic waves travel in the $x-y$ plane; thus, $\mathbf{H}$ and $\mathbf{E}$ are independent of $z$ in the equations. The equations are then decoupled into two independent sets of equations. The first set is

$$
\begin{gathered}
\frac{\partial E_{z}}{\partial y}=-\mu_{0} \frac{\partial H_{x}}{\partial t}, \\
\frac{\partial E_{z}}{\partial x}=\mu_{0} \frac{\partial H_{y}}{\partial t},
\end{gathered}
$$

$$
\frac{\partial H_{y}}{\partial x}-\frac{\partial H_{x}}{\partial y}=\varepsilon_{0} \varepsilon(\mathbf{x}) \frac{\partial E_{z}}{\partial t},
$$

and the second set is

$$
\begin{gathered}
\frac{\partial H_{z}}{\partial y}=\varepsilon_{0} \varepsilon(\mathbf{x}) \frac{\partial E_{x}}{\partial t}, \\
\frac{\partial H_{z}}{\partial x}=-\varepsilon_{0} \varepsilon(\mathbf{x}) \frac{\partial E_{y}}{\partial t}, \\
\frac{\partial E_{y}}{\partial x}-\frac{\partial E_{x}}{\partial y}=-\mu_{0} \frac{\partial H_{z}}{\partial t} .
\end{gathered}
$$

The first set is called $E$ polarization, where $\mathbf{E}$ is parallel to the $z$ axis and $\mathbf{H}$ is in the $x-y$ plane. The second set, on the other hand, is called $H$ polarization, where $\mathbf{H}$ is parallel to the $z$ axis and $\mathbf{E}$ is in the $x-y$ plane.

We derive first the eigenvalue equation of $E$ polarization. From the first set [Eqs. (8)-(10)], we obtain the wave equation in terms of $E_{z}$, 
(a)

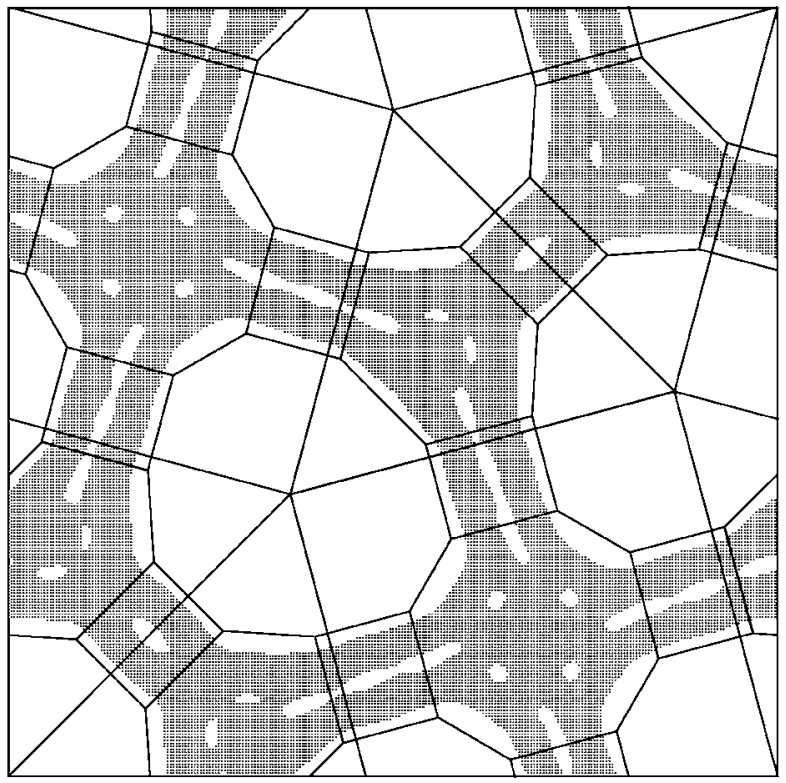

(b)

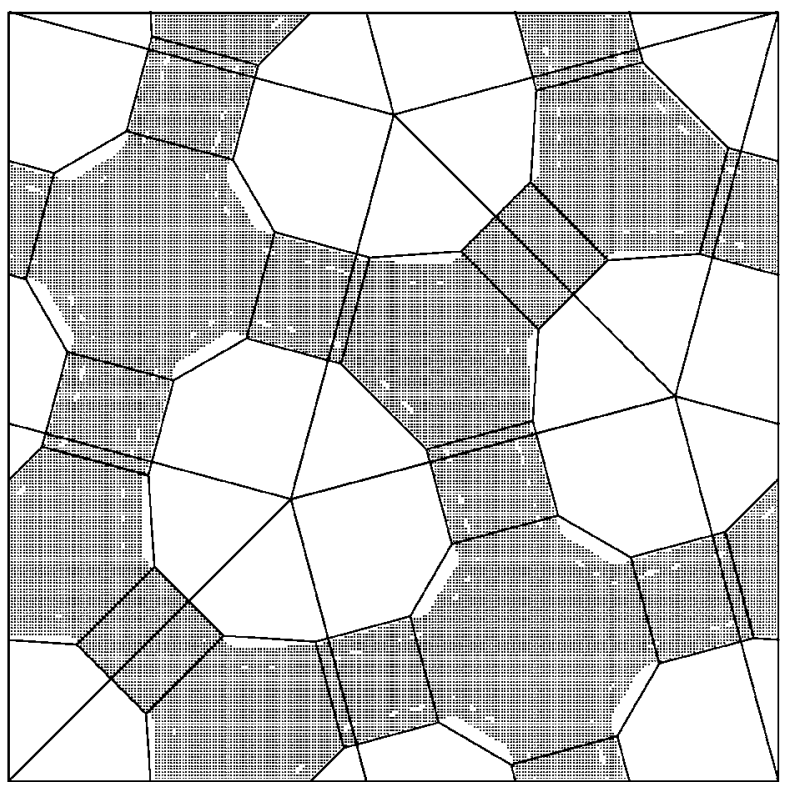

FIG. 7. Plots of the Fourier series expansions of $1 / \varepsilon(\mathbf{x})$ by means of (a) $l=11$ (529 waves) and (b) $l=30$ (3721 waves) for $\left(3^{2} .4 .3 .4\right)$ with dielectric constants $\varepsilon_{A}=13, \varepsilon_{B}=13$, and $\varepsilon_{C}=1$, where $l$ is the maximum integer of $\left|l_{1}\right|$ or $\left|l_{2}\right|$ in Eq. (4). Shaded regions indicate $1 / \varepsilon<0.13$.

$$
\frac{1}{\varepsilon(\mathbf{x})}\left\{\frac{\partial^{2}}{\partial x^{2}}+\frac{\partial^{2}}{\partial y^{2}}\right\} E_{z}=\frac{1}{c^{2}} \frac{\partial^{2}}{\partial t^{2}} E_{z},
$$

where $c$ is the speed of light. We seek the solution of the form $E_{z}(\mathbf{x}, t)=E_{0}(\mathbf{x}) e^{-i \omega t}$, where $\omega$ is the eigenangular frequency, and $E_{0}(\mathbf{x})$ are the eigenfunction of the wave equation. $E_{0}(\mathbf{x})$ satisfies the following eigenvalue equation:

$$
-\frac{1}{\varepsilon(\mathbf{x})}\left\{\frac{\partial^{2}}{\partial x^{2}}+\frac{\partial^{2}}{\partial y^{2}}\right\} E_{0}(\mathbf{x})=\frac{\omega^{2}}{c^{2}} E_{0}(\mathbf{x}) \text {. }
$$

We apply Bloch's theorem expressed by

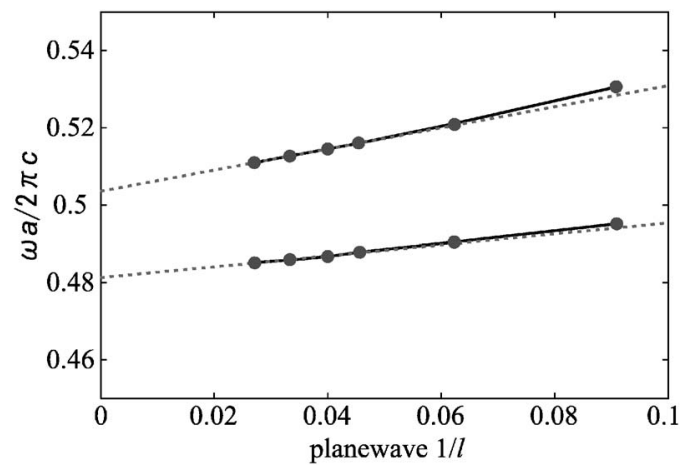

FIG. 8. Band gap as a function of $1 / l$ for $\left(3^{2} .4 .3 .4\right)$ with dielectric constants $\varepsilon_{A}=13, \varepsilon_{B}=13$, and $\varepsilon_{C}=1$, where $l$ is the maximum integer of $\left|l_{1}\right|$ or $\left|l_{2}\right|$ in Eq. (4). Upper points correspond to the bottom of the air band, and lower points correspond to the top of the dielectric band. From right to left, these points correspond to $l$ $=11,16,22,25,30$, and 37, namely, 529, 1089, 2025, 2601, 3721, and 5625 plane waves, respectively. These lines converge to 0.504 and 0.481 , indicating that the band gap remains.

$$
E_{0}(\mathbf{x})=u(\mathbf{x}) e^{i \mathbf{k} \cdot \mathbf{x}}
$$

where

$$
u\left(\mathbf{x}+\mathbf{a}_{i}\right)=u(\mathbf{x})
$$

and $E_{0}(\mathbf{x})$ can thus be represented by

$$
E_{0}(\mathbf{x})=\sum_{\mathbf{G}} \psi_{\mathbf{G}} e^{i(\mathbf{k}+\mathbf{G}) \cdot \mathbf{x}} .
$$

We finally obtain the eigenvalue equation for the expansion coefficients $\psi_{\mathbf{G}}$ as follows:

$$
\sum_{\mathbf{G}^{\prime}} \kappa\left(\mathbf{G}-\mathbf{G}^{\prime}\right)\left|\mathbf{k}+\mathbf{G}^{\prime}\right|^{2} \psi_{\mathbf{G}^{\prime}}=\frac{\omega^{2}}{c^{2}} \psi_{\mathbf{G}}
$$

The coefficient matrix is not Hermitian, and we introduce a new vector defined by

$$
\xi_{\mathbf{G}}=|\mathbf{k}+\mathbf{G}| \psi_{\mathbf{G}} .
$$

Using this vector, we obtain the eigenvalue equation with a Hermitian matrix:

$$
\sum_{\mathbf{G}^{\prime}} \kappa\left(\mathbf{G}-\mathbf{G}^{\prime}\right)|\mathbf{k}+\mathbf{G}|\left|\mathbf{k}+\mathbf{G}^{\prime}\right| \xi_{\mathbf{G}^{\prime}}=\frac{\omega^{2}}{c^{2}} \xi_{\mathbf{G}} .
$$

In the case of $H$ polarization, from the second set of equations [Eqs. (11)-(13)], we obtain the wave equation

$$
\left\{\frac{\partial}{\partial x} \frac{1}{\varepsilon(\mathbf{x})} \frac{\partial}{\partial x}+\frac{\partial}{\partial y} \frac{1}{\varepsilon(\mathbf{x})} \frac{\partial}{\partial y}\right\} H_{z}=\frac{1}{c^{2}} \frac{\partial^{2}}{\partial t^{2}} H_{z},
$$

and again we seek the solution of the form $H_{z}(\mathbf{x}, t)$ $=H_{0}(\mathbf{x}) e^{-i \omega t}$. Then, we obtain

$$
-\left\{\frac{\partial}{\partial x} \frac{1}{\varepsilon(\mathbf{x})} \frac{\partial}{\partial x}+\frac{\partial}{\partial y} \frac{1}{\varepsilon(\mathbf{x})} \frac{\partial}{\partial y}\right\} H_{0}(\mathbf{x})=\frac{\omega^{2}}{c^{2}} H_{0}(\mathbf{x}),
$$

where $H_{0}(\mathbf{x})$ is the eigenfunction of the wave equation.

The Bloch theorem allows us to express $H_{0}(\mathbf{x})$ as 


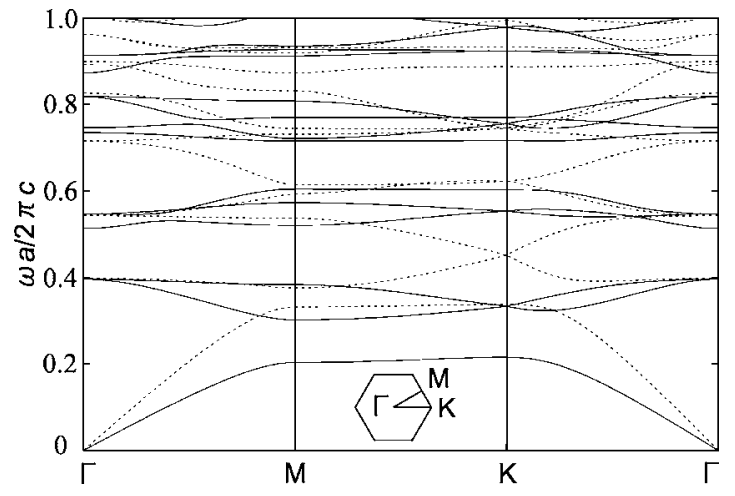

FIG. 9. Photonic band structure for $\left(6^{3}\right)$ with dielectric constants $\varepsilon_{A}=13, \varepsilon_{B}=1$, and $\varepsilon_{C}=1: E$ polarization (solid line) and $H$ polarization (broken line).

$$
H_{0}(\mathbf{x})=\sum_{\mathbf{G}} \varphi_{\mathbf{G}} e^{i(\mathbf{k}+\mathbf{G}) \cdot \mathbf{x}} .
$$

The eigenvalue equation with a Hermitian matrix in terms of $\varphi_{\mathbf{G}}$, which we should solve numerically, is

$$
\sum_{\mathbf{G}^{\prime}} \kappa\left(\mathbf{G}-\mathbf{G}^{\prime}\right)(\mathbf{k}+\mathbf{G}) \cdot\left(\mathbf{k}+\mathbf{G}^{\prime}\right) \varphi_{\mathbf{G}^{\prime}}=\frac{\omega^{2}}{c^{2}} \varphi_{\mathbf{G}} .
$$

Since Eqs. (19) and (25) are different, the band structures of $E$ and $H$ polarizations are different. We seek complete PBGs where the gaps of both $E$ and $H$ polarizations overlap.

\section{B. Accuracy of computation}

It is known that the computation of accurate twodimensional photonic band structures of complex patterns is nontrivial and requires a certain care. To check the convergence of eigenvalues and, consequently, to show the accuracy of our results, we first illustrate how Fourier series expansions reproduce the most complicated structure $\left(3^{2} .4 .3 .4\right)$, and second, we show how a band gap converges with changing the number of waves in the expansion in Eq. (3). We select $\left(3^{2} .4 .3 .4\right)$ because its lattice constant is larger than those of SJC patterns in the unit of edge length of polygons.

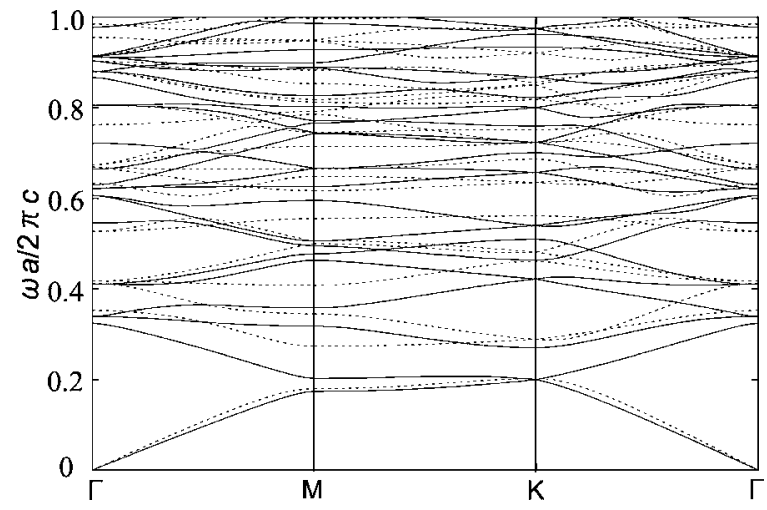

FIG. 10. Photonic band structure for $\left(6^{3}\right)$ with dielectric constants $\varepsilon_{A}=1, \varepsilon_{B}=13$, and $\varepsilon_{C}=13: E$ polarization (solid line) and $H$ polarization (broken line).

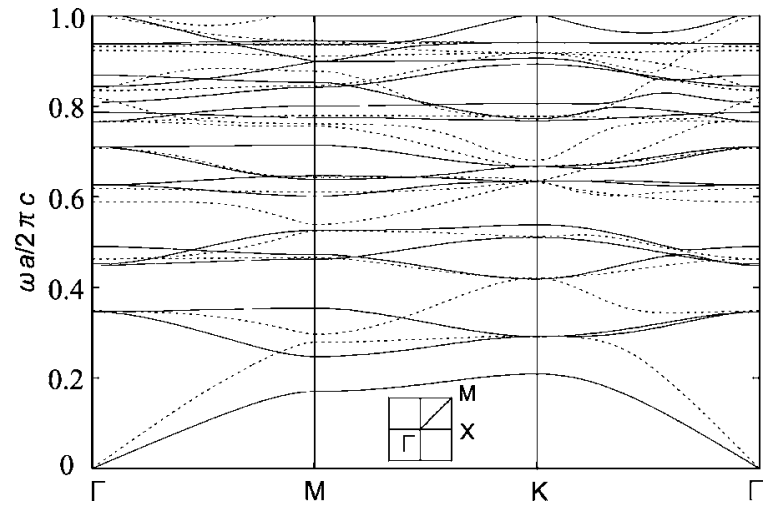

FIG. 11. Photonic band structure for $\left(4.8^{2}\right)$ with dielectric constants $\varepsilon_{A}=13, \varepsilon_{B}=1$, and $\varepsilon_{C}=1: E$ polarization (solid line) and $H$ polarization (broken line).

Suppose $-l \leqslant l_{i} \leqslant l$, where $l_{i}$ is $l_{1}$ or $l_{2}$ in Eq. (4). The number of plane waves is $(2 l+1)^{2}$. We have checked the Fourier coefficient in Eq. (5) by reproducing $1 / \varepsilon(\mathbf{x})$. The integral Eq. (5) is computed through 256 mesh points in each direction. Figure 7 shows plots of the Fourier series expansions for a $\left(3^{2} .4 .3 .4\right)$ structure with dielectric constants $\varepsilon_{A}$ $=13, \varepsilon_{B}=13$, and $\varepsilon_{C}=1$. In Fig. 7(a), there is a substantial amount of large islands. Consequently, it implies that the accuracy of band structures using 529 waves is dubious. On the contrary, the higher expansion by 3721 waves reproduces well the complex pattern as shown in Fig. 7(b).

Figure 8 shows the largest band gap of $E$ polarization as a function of $1 / l$ for the same structure (Fig. 17). Upper points correspond to the bottom of the air band, and lower points correspond to the top of the dielectric band. From right to left, these points correspond to 529, 1089, 2025, 2601, 3721, and 5625 plane waves, respectively.

Broken lines are least-squares fits to four points with larger $l$. The extrapolation to values with $1 / l=0$ demonstrates that the band gap remains. As far as we have done, the topology of generic band structures (not in detail) for 529 and 3721 waves give the same one for all patterns. To obtain more accurate values as possible, we have chosen 3721 plane waves. Below all results, both figures and values are obtained using 3721 waves.

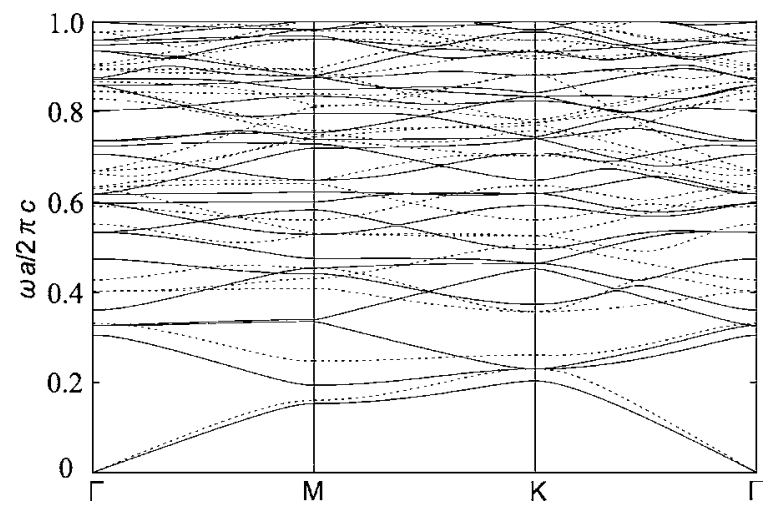

FIG. 12. Photonic band structure for $\left(4.8^{2}\right)$ with dielectric constants $\varepsilon_{A}=1, \varepsilon_{B}=13$, and $\varepsilon_{C}=13: E$ polarization (solid line) and $H$ polarization (broken line). 


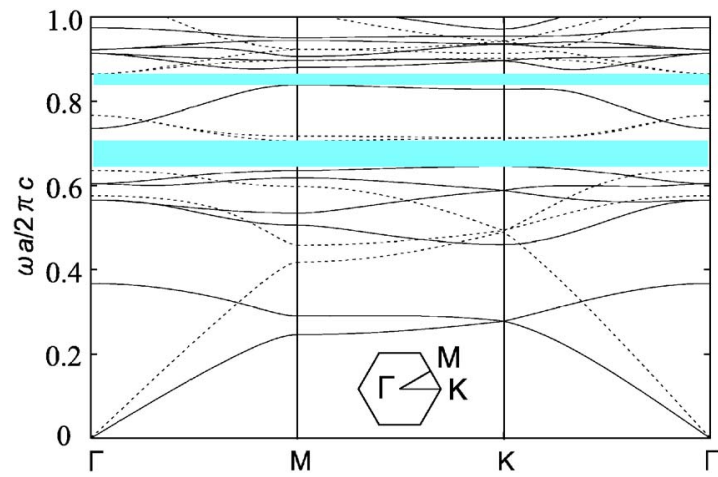

FIG. 13. (Color online) Photonic band structure for (4.6.12) with dielectric constants $\varepsilon_{A}=13, \varepsilon_{B}=1$, and $\varepsilon_{C}=1: E$ polarization (solid line) and $H$ polarization (broken line). The gap-midgap ratios $(\Delta \omega / \omega)$ are $9.3 \times 10^{-2}$ (bottom) and $3.0 \times 10^{-2}$ (top).

\section{PHOTONIC BAND STRUCTURES}

Before going into details of band structures, let us remind readers of two generic tendencies about how PBGs open, which depend on the vector nature of waves and air-filling factor of dielectric structures. Then, we present band structure calculations for Archimedean tiling patterns.

\section{A. Dielectric cylinders and air cylinders}

Making the dielectric contrast by $\varepsilon_{\mathrm{air}}=1$ (air) and $\varepsilon_{\mathrm{di}}$ $=13$, which are usually used in the literature, we have investigated all combinations of dielectric constant, i.e., two types of photonic crystals for the $\left(6^{3}\right)$ structure, four types for the $\left(4.8^{2}\right)$ structure, and six types for (4.6.12) and $\left(3^{2} .4 .3 .4\right)$ structures. The positions of components $A, B$, and $C$ are defined as in Figs. 5 and 6.

There are two types of dielectric structures in two dimensions: dielectric cylinders and air cylinders. Dielectric cylinders imply a structure composed of isolated high-dielectricconstant cylinders. Air cylinders are isolated low-dielectricconstant ones; in other words, the structure is a connected high-dielectric-constant vein.

The vector nature is a key difference from electronic problems, which are governed by the scalar Schrödinger

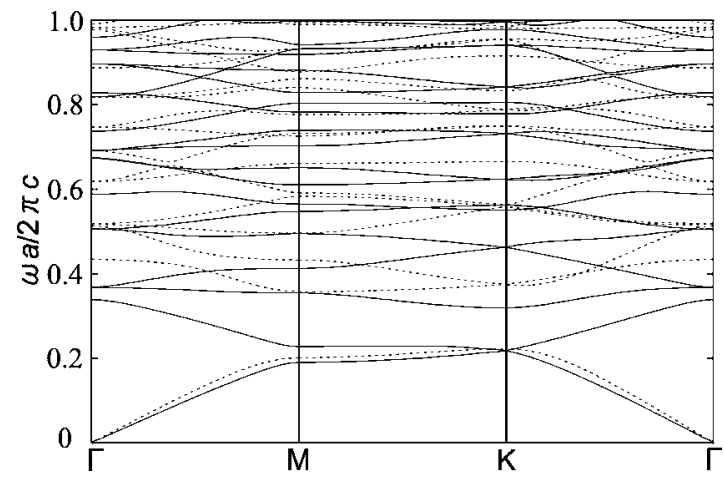

FIG. 14. Photonic band structure for (4.6.12) with dielectric constants $\varepsilon_{A}=13, \varepsilon_{B}=13$, and $\varepsilon_{C}=1: E$ polarization (solid line) and $H$ polarization (broken line).

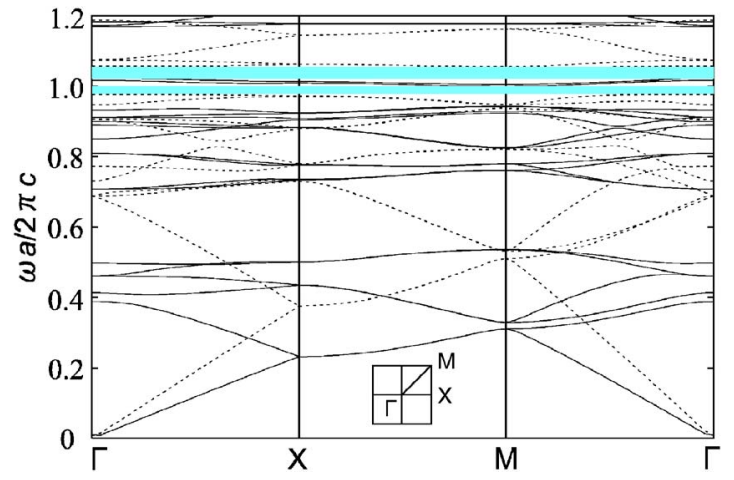

FIG. 15. (Color online) Photonic band structure for $\left(3^{2}\right.$.4.3.4) with dielectric constants $\varepsilon_{A}=13, \varepsilon_{B}=1$, and $\varepsilon_{C}=1$ : $E$ polarization (solid line) and $H$ polarization (broken line). The gap-midgap ratios $(\Delta \omega / \omega)$ are $2.2 \times 10^{-2}$ (bottom) and $3.1 \times 10^{-2}$ (top).

wave equation. It is well known that dielectric cylinders tend to have PBGs with $E$ polarization, and that air cylinders tend to have PBGs with $H$ polarization. ${ }^{17}$

Electromagnetic energy is reduced when the displacement field $D$ can localize in regions with higher dielectric constant. The corresponding states form the first band (dielectric band). When the displacement field is excluded from higherdielectric regions, the energy increases and, accordingly, the corresponding states form the second band (air band). Between these two bands, we have a PBG.

For dielectric cylinders, displacement fields of $E$ polarization can concentrate into isolated high-dielectric regions, while the displacement fields of $H$ polarization cannot be localized on isolated regions because of the transverse continuity of the vector fields. On the contrary, for air cylinders, the displacement fields of $H$ polarization can continuously extend to networks with high-dielectric constant, leading to the reduction of energy.

\section{B. Air-filling factor and dielectric fluctuations}

The air-filling factor is another important parameter. Generally, strong dielectric contrast makes PBGs wider. It has been argued that dielectric fluctuations provide a guide to

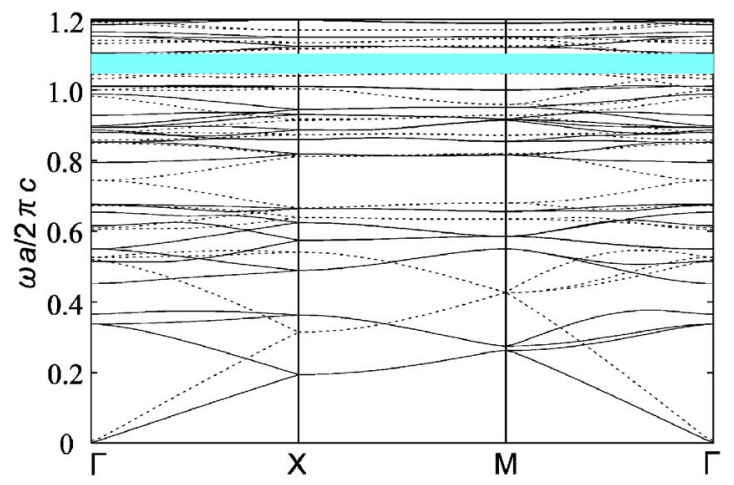

FIG. 16. (Color online) Photonic band structure for $\left(3^{2} \cdot 4.3 .4\right)$ with dielectric constants $\varepsilon_{A}=1, \varepsilon_{B}=1$, and $\varepsilon_{C}=13: E$ polarization (solid line) and $H$ polarization (broken line). The gap-midgap ratio $(\Delta \omega / \omega)$ is $5.1 \times 10^{-2}$. 
have a better choice of the air-filling factor. ${ }^{19}$ The average of dielectric constant and the fluctuation are

$$
\begin{gathered}
\bar{\varepsilon}=f \varepsilon_{\mathrm{air}}+(1-f) \varepsilon_{\mathrm{di}}, \\
(\Delta \varepsilon)^{2}=\frac{f \varepsilon_{\mathrm{air}}^{2}+(1-f) \varepsilon_{\mathrm{di}}^{2}}{\left(f \varepsilon_{\mathrm{air}}+(1-f) \varepsilon_{\mathrm{di}}\right)^{2}}-1,
\end{gathered}
$$

where $f$ is the air-filling factor. For a given choice of dielectric contrasts, the fluctuation has a maximum for a filling factor as follows:

$$
f_{\max }=\frac{\varepsilon_{\mathrm{di}}}{\varepsilon_{\mathrm{air}}+\varepsilon_{\mathrm{di}}} .
$$

It has been argued that this maximum gives the strongest scattering of electromagnetic waves. In our case of $\varepsilon_{\text {air }}=1$ and $\varepsilon_{\mathrm{di}}=13$, the better choice of the air-filling factor is about 93\%. In practice, the higher the air-filling factor is, the larger the PBG is. This argument may be useful when one designs the ratio of components of a block copolymer before exact band calculations are at hand. It should be mentioned that the argument is independent of structures, dimensions, and the degree of ordering, and that it is accordingly by no means accurate.

\section{C. $\left(6^{3}\right)$ structure}

We present photonic band structures for a $\left(6^{3}\right)$ tiling with an area ratio of $A: B: C=1: 1: 1$. The plane group is $p 6 \mathrm{~mm}$. Dielectric constants are $\varepsilon_{A}=13, \varepsilon_{B}=1$, and $\varepsilon_{C}=1$ in the case of dielectric cylinders (Fig. 9), and $\varepsilon_{A}=1, \varepsilon_{B}=13$, and $\varepsilon_{C}$ $=13$ in the case of air cylinders (Fig. 10). Dielectric cylinders have broad PBGs in $E$ polarization, and narrow ones in $H$ polarization, but their positions are different. Thus, there is no complete PBG. Air cylinders have PBGs in $H$ polarization, and very narrow one in $E$ polarization.

It is known that a triangular lattice composed of air cylinders with larger fractions of air has the complete PBG. ${ }^{15}$ However, the air-filling factor of the $\left(6^{3}\right)$ is $33 \%$, which is too small to obtain the complete PBG.

\section{D. $\left(4.8^{2}\right)$ structure}

We present photonic band structures for a $\left(4.8^{2}\right)$ tiling with an area ratio of $A: B: C=2: 1: 2$. The plane group is $p 4 \mathrm{~mm}$. There are two ways to construct dielectric cylinders: $\varepsilon_{A}=13, \varepsilon_{B}=1, \varepsilon_{C}=1$ (Fig. 11) and $\varepsilon_{A}=1, \varepsilon_{B}=13, \varepsilon_{C}=1$. The former has large PBGs in $E$ polarization and a narrow PBG in $H$ polarization. These band gaps are very close but do not overlap. The latter has PBGs only in $E$ polarization. Similarly, there are two ways to construct air cylinders: $\varepsilon_{A}=1$, $\varepsilon_{B}=13, \varepsilon_{C}=13$ (Fig. 12) and $\varepsilon_{A}=13, \varepsilon_{B}=1, \varepsilon_{C}=13$. Only in Fig. 12 we see PBGs in $H$ polarization. In any case, there is no complete PBG in the $\left(4.8^{2}\right)$ tiling. We think that these results are essentially the same as the results of about $40 \%$ or $20 \%$ cylinders on square lattices.

\section{E. (4.6.12) structure}

We present photonic band structures for a (4.6.12) tiling with an area ratio of $A: B: C=1: 1: 2$. The plane group is

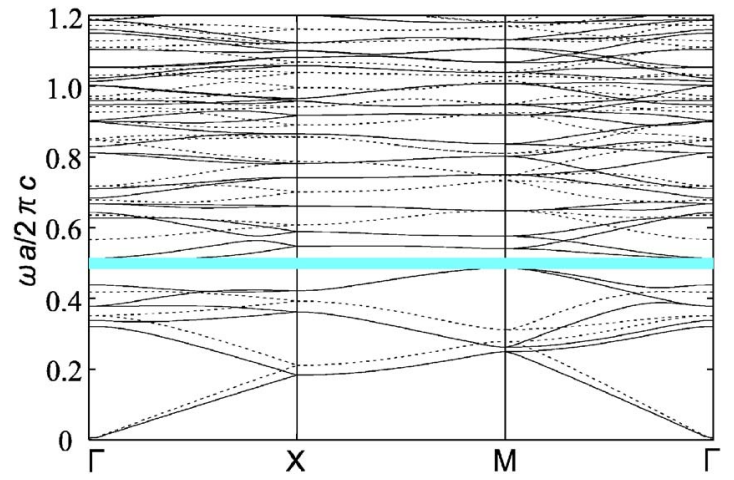

FIG. 17. (Color online) Photonic band structure for $\left(3^{2}\right.$.4.3.4) with dielectric constants $\varepsilon_{A}=13, \varepsilon_{B}=13$, and $\varepsilon_{C}=1: E$ polarization (solid line) and $H$ polarization (broken line). The gap-midgap ratio $(\Delta \omega / \omega)$ is $5.4 \times 10^{-2}$.

$p 6 \mathrm{~mm}$. There are three ways to construct dielectric cylinders: $\varepsilon_{A}=13, \varepsilon_{B}=1, \varepsilon_{C}=1$ (Fig. 13), $\varepsilon_{A}=1, \varepsilon_{B}=13, \varepsilon_{C}=1$, and $\varepsilon_{A}=1, \varepsilon_{B}=1, \varepsilon_{C}=13$. All cases have large PBGs in waves of $E$ polarization, and $A$ and $C$ dielectric cylinders have PBGs in waves of $H$ polarization. More importantly, in the case of $A$ dielectric cylinders, there are complete PBGs with $75 \%$ air-filling factor. The structure looks like that composed of $C$ air cylinders. Let $\omega$ be the frequency at the middle of a band gap and $\Delta \omega$ be the gap frequency width. The scale independent gap-midgap ratios $(\Delta \omega / \omega)$ are $9.3 \times 10^{-2}$ and 3.0 $\times 10^{-2}$.

On the other hand, there are three ways to construct air cylinders: $\varepsilon_{A}=1, \varepsilon_{B}=13, \varepsilon_{C}=13 ; \varepsilon_{A}=13, \varepsilon_{B}=1, \varepsilon_{C}=13$; and $\varepsilon_{A}=13, \varepsilon_{B}=13, \varepsilon_{C}=1$ (Fig. 14). All cases have large PBGs in waves of $H$ polarization. $B$ and $C$ air cylinders have $E$ PBGs as well, but there is no complete PBGs.

The $C$ air cylinders form the triangular lattice. However, like the case of $\left(6^{3}\right)$, the fraction of the $C$ air cylinders cannot be far beyond 0.5 , and the air cylinders do not show complete PBGs (Fig. 14). Increasing the fraction of $C$ component, which is necessary for complete PBGs, ${ }^{15,17}$ will make the structure undergo a microphase separation to another structure. Instead of enlarging $C$ air cylinders, $A$ dielectric

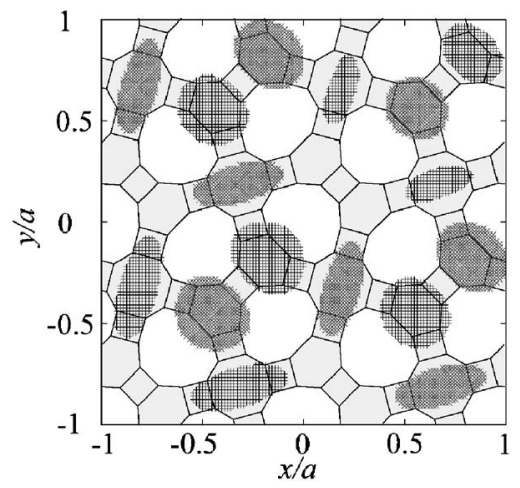

FIG. 18. Electric field of near $M$-point $E$ polarization mode in the dielectric band for $\left(3^{2} .4 .3 .4\right)$ with dielectric constants $\varepsilon_{A}=13$, $\varepsilon_{B}=13$, and $\varepsilon_{C}=1$. Different shades indicate the plus and minus of the field. The field mainly concentrates into $A$ regions, leading to the reduction of energy. 


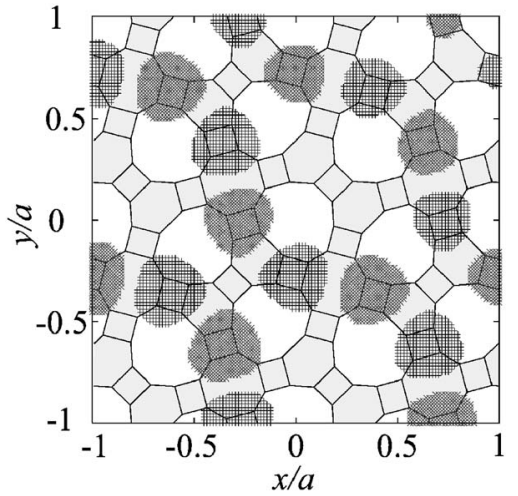

FIG. 19. Electric field of near $M$-point $E$ polarization mode in the air band for $\left(3^{2} .4 .3 .4\right)$ with dielectric constants $\varepsilon_{A}=13, \varepsilon_{B}$ $=13$, and $\varepsilon_{C}=1$. Different shades indicate the plus and minus of the field. The field mainly concentrates into $B$ regions; however, it invades $C$ air cylinders more and thus increases the displacement energy.

cylinders forming the honeycomb lattice is promising as shown in Fig. 13. The fact that the honeycomb lattice has the complete PBG has been known. ${ }^{16,17}$ In this case, since $B$ regions do not have the high dielectric constant, a gap of $H$ polarization around 0.3 seen in Fig. 14 disappears.

\section{F. $\left(3^{2} \cdot 4.3 .4\right)$ structure}

We present photonic band structures for a $\left(3^{2} .4 .3 .4\right)$ tiling with an area ratio of $A: B: C=9: 7: 14$. The plane group is $p 4 \mathrm{gm}$, which is a nonsymmorphic group leading to the sticking together of bands. There are three ways to construct dielectric cylinders: $\varepsilon_{A}=13, \varepsilon_{B}=1, \varepsilon_{C}=1$ (Fig. 15); $\varepsilon_{A}=1, \varepsilon_{B}$ $=13, \varepsilon_{C}=1$; and $\varepsilon_{A}=1, \varepsilon_{B}=1, \varepsilon_{C}=13$ (Fig. 16). All cases have large $\mathrm{PBG}$ in waves of $E$ polarization and $H$ polarization. More importantly, in the case of $A$ and $C$ dielectric cylinders, they have complete PBGs. The centers of these PBGs are at almost the same position. The gap-midgap ratios $(\Delta \omega / \omega)$ for $A$ dielectric cylinders are $2.2 \times 10^{-2}$ and 3.1 $\times 10^{-2}$, and that of $C$ ones is $5.1 \times 10^{-2}$.

On the other hand, there are three ways to construct air cylinders: $\varepsilon_{A}=1, \varepsilon_{B}=13, \varepsilon_{C}=13 ; \varepsilon_{A}=13, \varepsilon_{B}=1, \varepsilon_{C}=13$; and $\varepsilon_{A}=13, \varepsilon_{B}=13, \varepsilon_{C}=1$ (Fig. 17). All cases have PBGs in waves of $H$ polarization. In Fig. 17, there are again complete PBGs. The center of the PBG in this case is at almost half compared to the case of dielectric cylinders. It is quite interesting that both $C$ dielectric and $C$ air cylinders of the same structure have complete PBGs. The gap-midgap ratio $(\Delta \omega / \omega)$ for $C$ air cylinders is $5.4 \times 10^{-2}$. When we increase air-filling factor up to $50 \%(A: B: C=9: 7: 16)$, the gapmidgap ratio becomes $6.6 \times 10^{-2}$.

We show the electric fields with $E$ polarization for $C$ air cylinders with respect to the gap $(\omega a / 2 \pi c \sim 0.5)$ in Fig. 17.

TABLE II. Summary of the photonic band structures. Structures are dielectric cylinders (D) with $\varepsilon_{\alpha}$ $=13$ and $\varepsilon_{\beta}=\varepsilon_{\gamma}=1$ or air cylinders (A) with $\varepsilon_{\alpha}=1$ and $\varepsilon_{\beta}=\varepsilon_{\gamma}=13$, where $\alpha, \beta$, and $\gamma$ stand for combinations of $A, B$, and $C$. In the case of $\left(3^{2} .4 .3 .4\right), C^{*}$ implies data for $A 9 B 7 C 16$. In the fourth column, $E$ or $H$ means its polarization has the largest PBG. In the second to the last column, when complete PBGs exist, the gap-midgap ratios of the complete PBGs $(\Delta \omega / \omega)$ are listed, where $\omega$ is the eigenangular frequency at the middle of a gap and $\Delta \omega$ is the gap width.

\begin{tabular}{|c|c|c|c|c|c|}
\hline Tiling & Cylinder & $\alpha$ & Polarization & $\Delta \omega / \omega$ & Fig \\
\hline$\left(6^{3}\right)$ & $\mathrm{D}$ & A & $E$ & & 9 \\
\hline p6mm & A & A & $H$ & & 10 \\
\hline$\left(4.8^{2}\right)$ & $\mathrm{D}$ & A & $E$ & & 11 \\
\hline \multirow[t]{3}{*}{$p 4 m m$} & & B & $E$ & & \\
\hline & A & A & $H$ & & 12 \\
\hline & & B & & & \\
\hline$(4.6 .12)$ & $\mathrm{D}$ & A & $E$ & $0.093,0.030$ & 13 \\
\hline \multirow[t]{5}{*}{ p6mm } & & B & $E$ & & \\
\hline & & $\mathrm{C}$ & $E$ & & \\
\hline & A & A & $H$ & & \\
\hline & & B & $H$ & & \\
\hline & & $\mathrm{C}$ & $H$ & & 14 \\
\hline$\left(3^{2} \cdot 4 \cdot 3 \cdot 4\right)$ & $\mathrm{D}$ & A & $E$ & $0.022,0.031$ & 15 \\
\hline \multirow[t]{6}{*}{$p 4 g m$} & & B & $E$ & & \\
\hline & & $\mathrm{C}$ & $E$ & 0.051 & 16 \\
\hline & A & A & $H$ & & \\
\hline & & B & $H$ & & \\
\hline & & $\mathrm{C}$ & $H$ & 0.054 & 17 \\
\hline & & $\mathrm{C}^{*}$ & $H$ & 0.066 & \\
\hline
\end{tabular}




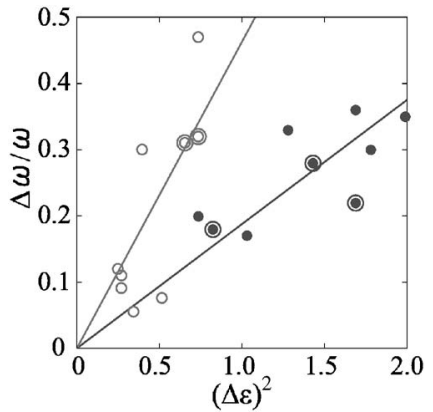

FIG. 20. The largest gap-midgap ratio against dielectric fluctuations: $E$ polarization of dielectric cylinders (solid circle) and $H$ polarization of air cylinders (open circle) are plotted for $\left(6^{3}\right),\left(4.8^{2}\right)$, (4.6.12), and $\left(3^{2} \cdot 4.3 .4\right)$. Double circles correspond to the cases of complete photonic band gaps. The least-squares fit is estimated from solid or open data points; the slope is 0.19 or 0.46 , respectively.

An eigenfunction of the dielectric band at a point near the $M$ point a little way off to the $\Gamma$ point is displayed in Fig. 18. The field concentrates mainly into $A$ regions. The eigenfunction of the same point at the bottom of the air band is shown in Fig. 19. The field is mainly concentrated into $B$ regions; however, it invades $C$ air cylinders more and thus increases the displacement energy.

\section{DISCUSSION AND SUMMARY}

We have investigated photonic band structures of twodimensional Archimedean tiling patterns, $\left(4.8^{2}\right),\left(6^{3}\right)$, (4.6.12), and $\left(3^{2} .4 .3 .4\right)$, observed in self-assembled microphase separation of $A B C$ star block terpolymer systems. In Table II, we summarize the results. We call attention that the honeycomb lattice obtained from the (4.6.12) structure, in fact, has complete PBGs. Moreover, it is remarkable that three types of the $\left(3^{2} .4 .3 .4\right)$ structures favor complete PBGs.

As discussed in Sec. V, there is a clear tendency in Table II that dielectric cylinders tend to have PBGs with $E$ polarization and that air cylinders tend to have PBGs with $H$ polarization. Furthermore, there are correlations between the dielectric fluctuations defined by Eq. (27) and the gapmidgap ratio of $E$ polarization of dielectric cylinders and that of $H$ polarization of air cylinders, see Fig. 20. This plot displays the gap-midgap ratio of the largest band gap for $E$ polarization of dielectric cylinders (solid circle) and $H$ polarization of air cylinders (open circle). With increasing the dielectric fluctuations, the ratio for both polarizations increases. However, it appears that the existence of complete
PBGs (double circles) has nothing to do with the dielectric fluctuations. Of course, when a complete band gap exists for a lattice, the ratio correlates with the fluctuation.

Triangular lattices have been extensively studied in the case of hexagonal phases of $A B$ diblock and $A B A$ triblock copolymers. ${ }^{20}$ The area ratio of cylinders in the traditional linear polymers is less than about $30 \%$, which is an inevitable drawback by using these polymers to construct air cylinders with high air-filling factors. In contrast, the (4.6.12) structure provides fascinating possibility: Instead of constructing air cylinders with large radius, the dielectric $A$ cylinders of (4.6.12) forming the honeycomb lattice could be a good substitute. The phase region of (4.6.12) in the phase diagram (Fig. 2) is wide, and it is thus easy to form the structure. This is one of feasible routes to self-assemble the 2D soft photonic crystals with complete PBGs.

We have found that in both dielectric and air cases, PBGs easily open in the (32.4.3.4) Archimedean tiling structures. It should be noticed that both dielectric and air cylinders with the same shape have PBGs. Furthermore, for $A 9 B 7 C 16$ with air-filling factor of 50\%, the gap-midgap ratio for $C$ air cylinders becomes 0.066 . As mentioned in the Introduction, the $\left(3^{2}\right.$.4.3.4) is akin to the dodecagonal quasicrystals: our ongoing study will clarify the difference between the band structure of $\left(3^{2} .4 .3 .4\right)$ and that of the higher approximant of dodecagonal quasicrystals.

Polymers have the dielectric constant of $\varepsilon \sim 2$. We have calculated the case with the dielectric contrast that is equal to 2 and have found that there are no PBGs in the ( $\left.3^{2} .4 .3 .4\right)$ with composition $A 9 B 7 C 14$.

To make high dielectric contrast, selective deposition of CdSe nanocrystals to the 2-vinylpyridine microdomains and selective removal of isoprene microdomains by UV etching treatment or ozonolysis have been developed. ${ }^{20,21}$ Actually, polyisoprene and poly(2-vinylpyridine) have been already used in experiments. ${ }^{5,6}$ An alternative way is by using organic-inorganic nanocomposite, which has been constructed as well. ${ }^{48}$

In conclusion, the bottom-up complex Archimedean tilings such as (4.6.12) and $\left(3^{2}\right.$.4.3.4) offer a way to construct photonic crystals. Subtle changes of the symmetry, shape, and fraction of dielectric structures affect band structures. Further study to optimize PBGs will be necessary.

\section{ACKNOWLEDGMENTS}

We are grateful to Y. Matsushita and A. Takano for helpful discussions and to K. Edagawa and J. Matsuzawa for useful comments. We are indebted to the University of the Air for using computing facilities and to the Ogasawara Science and Technology Foundation for financial support.
${ }^{1}$ B. Grünbaum and G. C. Shephard, Tilings and Patterns (Freeman, New York, 1986).

${ }^{2}$ T. Gemma, A. Hatano, and T. Dotera, Macromolecules 35, 3225 (2002).
${ }^{3}$ T. Dotera and A. Hatano, J. Chem. Phys. 105, 8413 (1996).

${ }^{4}$ Y. Bohbot-Raviv and Z.-G. Wang, Phys. Rev. Lett. 85, 3428 (2000).

${ }^{5}$ A. Takano, S. Wada, S. Sato, T. Araki, K. Hirahara, T. Kazama, S. 
Kawahara, Y. Isono, A. Ohno, N. Tanaka, and Y. Matsushita, Macromolecules 37, 9941 (2004).

${ }^{6}$ A. Takano, W. Kawashima, A. Noro, Y. Isono, N. Tanaka, T. Dotera, and Y. Matsushita, J. Polym. Sci., Part B: Polym. Phys. 43, 2427 (2005).

${ }^{7}$ H. Hückstädt, A. Göpfert, and V. Abetz, Macromol. Chem. Phys. 201, 296 (2000).

${ }^{8}$ K. Yamauchi, K. Takahashi, H. Hasegawa, H. Iatrou, N. Hadjichristidis, T. Kaneko, Y. Nishikawa, H. Jinnai, T. Matsui, H. Nishioka, M. Shimizu, and H. Furukawa, Macromolecules 36, 6962 (2003).

${ }^{9}$ S. Sioula, N. Hadjichristidis, and E. L. Thomas, Macromolecules 31, 5272 (1998); 31, 8429 (1998).

${ }^{10}$ K. Ohtaka, Phys. Rev. B 19, 5057 (1979).

${ }^{11}$ E. Yablonovitch, Phys. Rev. Lett. 58, 2059 (1987).

${ }^{12}$ S. John, Phys. Rev. Lett. 58, 2486 (1987).

${ }^{13}$ K. M. Ho, C. T. Chan, and C. M. Soukoulis, Phys. Rev. Lett. 65, 3152 (1990).

${ }^{14}$ M. Plihal and A. A. Maradudin, Phys. Rev. B 44, 8565 (1991).

${ }^{15}$ P. R. Villeneuve and M. Piché, Phys. Rev. B 46, 4969 (1992).

${ }^{16}$ C. M. Anderson and K. P. Giapis, Phys. Rev. Lett. 77, 2949 (1996).

${ }^{17}$ J. D. Joannopolous, R. D. Meade, and J. N. Winn, Photonic Crystals (Princeton University, Princeton, 1995). The complete PBG of the honeycomb lattice of dielectric cylinders is given in $\mathrm{p}$. 125.

${ }^{18}$ K. Sakoda, Optical Properties of Photonic Crystals (SpringerVerlag, Berlin, 2001).

${ }^{19}$ H. S. Sözüer, J. W. Haus, and R. Inguva, Phys. Rev. B 45, 13962 (1992).

${ }^{20}$ C. Park, J. Yoon, and E. L. Thomas, Polymer 44, 6725 (2003).

${ }^{21}$ Y. Fink, A. M. Urbas, M. G. Bawendi, J. D. Joannopoulos, and E. L. Thomas, J. Lightwave Technol. 17, 1963 (1999).

${ }^{22}$ I. W. Hamley, The Physics of Block Copolymers (Oxford University Press, New York, 1998).

${ }^{23}$ F. S. Bates and G. H. Fredrickson, Phys. Today 52(2), 32 (1999).

${ }^{24}$ N. Hadjichristidis, S. Pipas, and G. Floudas, Block Copolymers: Synthetic Strategies, Physical Properties, and Applications (Wiley, Hoboken, NJ, 2003).

${ }^{25}$ V. Z.-H. Chan, J. Hoffman, V. Y. Lee, H. Iatrou, A. Avgeropoulos, N. Hadjichristidis, R. D. Miller, and E. L. Thomas, Science 286, 1716 (1999).

${ }^{26}$ L. Martín-Moreno, F. J. García-Vidal, and A. M. Somoza, Phys. Rev. Lett. 83, 73 (1999).
${ }^{27}$ M. Maldovan, A. M. Urbas, N. Yufa, W. C. Carter, and E. L. Thomas, Phys. Rev. B 65, 165123 (2002).

${ }^{28}$ T. Dotera, Phys. Rev. Lett. 89, 205502 (2002).

${ }^{29}$ V. Babin, P. Garstecki, and R. Hołyst, Phys. Rev. B 66, 235120 (2002).

${ }^{30}$ K. Michielsen and J. S. Kole, Phys. Rev. B 68, 115107 (2003).

${ }^{31}$ Single junction class is the abbreviation of single junction point line class in Ref. 2. See Theorem 4.2 in the paper.

${ }^{32}$ T. Dotera, Phys. Rev. Lett. 82, 105 (1999). Another even polygon theorem appears in polyhedron packing in $A B C D$ star polymer systems.

${ }^{33}$ T. Ishimasa, H.-U. Nissen, and Y. Fukano, Phys. Rev. Lett. 55, 511 (1985).

${ }^{34}$ H. Chen, D. X. Li, and K. H. Kuo, Phys. Rev. Lett. 60, 1645 (1988).

${ }^{35}$ M. Oxborrow and C. L. Henley, Phys. Rev. B 48, 6966 (1993).

${ }^{36}$ The Physics of Quasicrystals, edited by P. J Steinhardt and S. Ostlund (World Scientific, Hackensack, NJ, 1987); Quasicrystals: The State of Art, edited by D. P. Divincenzo and P. J. Steinhardt (World Scientific, Hackensack, NJ, 1991).

${ }^{37}$ M. E. Zoorob, M. D. B. Charlton, G. J. Paker, J. J. Baumberg, and M. C. Netti, Nature (London) 404, 740 (2000); Mater. Sci. Eng., B 74, 168 (2000).

${ }^{38}$ S. David, A. Chelnokov, and J.-M. Lourtioz, IEEE J. Quantum Electron. 37, 1427 (2001).

${ }^{39}$ B. P. Hiett, D. H. Beckett, S. J. Cox, J. M. Generowicz, M. Molinari, and K. S. Thomas, J. Mater. Sci.: Mater. Electron. 14, 413 (2003).

${ }^{40}$ X. Zhang, Z.-Q. Zhang, and C. T. Chan, Phys. Rev. B 63, 081105(R) (2001).

${ }^{41}$ R. C. Gauthier and K. Mnaymneh, Opt. Express 13, 1985 (2005).

${ }^{42}$ Y. Wang, Y. Wang, S. Feng, and Z. Y. Li, Europhys. Lett. 74, 49 (2006).

${ }^{43}$ M. Notomi, H. Suzuki, T. Tamamura, and K. Edagawa, Phys. Rev. Lett. 92, 123906 (2004).

${ }^{44}$ W. Man, M. Megens, P. J. Steinhardt, and P. Chaikin, Nature (London) 436, 993 (2005).

${ }^{45}$ T. Dotera and T. Gemma, Philos. Mag. 86, 1085 (2006).

${ }^{46}$ T. Dotera, Philos. Mag. (to be published).

${ }^{47}$ K. Hayashida, T. Dotera, A. Takano, and Y. Matsushita, Phys. Rev. Lett. 98, 195502 (2007).

${ }^{48}$ A. C. Finnefrock, R. Ulrich, A. Du Chesne, C. C. Honeker, K. Schumacher, S. M. Gruner, U. Wiesner, and K. K. Unger, Angew. Chem., Int. Ed. 40, 1207 (2001). 